\title{
New closed-form proximity-effect complex permeability expression for characterizing Litz-Wire windings
}

\author{
Korawich Niyomsatian $^{*, \dagger}$, Johan. Gyselinck ${ }^{\dagger}$, and Ruth V. Sabariego* \\ ${ }^{*}$ EnergyVille, KU Leuven, Belgium, ${ }^{\dagger}$ BEAMS, Université Libre de Bruxelles, Belgium
}

Keywords: Eddy currents, proximity effect, litz-wire windings, homogenization, complex permeability

\begin{abstract}
The eddy current losses in litz-wire windings are mostly due to proximity effects, effects that can be macroscopically modelled via a complex frequency-dependent reluctivity. In this digest, we propose a new closed-form expression for this complex reluctivity. Results are compared with approximations found in the litterature.
\end{abstract}

\section{Introduction}

Accurately considering eddy-current effects in the winding of electromagnetic devices working at higher and higher frequencies implies the fine discretisation of each turn, which is unworkable in practice, particularly in litz-wire windings. With the advent of wide-bandgap semiconductors, able to operate at even higher frequencies, litz wires are increasingly present in e.g. switch mode supplies as they reduce the skin-effect losses to a minimum. At the strand level, the proximity-effect losses cannot be neglected, though [1].

Frequency-domain homogenization techniques provide a closed-form continuous representation of the homogenized winding, see e.g. [2,3] and references herein. Eddy-current effects are accounted for by a complex impedance in the supply electrical circuit (skin effect) and a complex reluctivity in the homogenized winding window (proximity effect). These two frequency-dependent complex parameters can be identified by means of an elementary 2D finite element (FE) model, as proposed in [4]. The approach has been successfully applied to litz-wire windings in [5].

In this work, we present an alternative closed-form representation of the proximity-effect complex permeabilily. The values obtained by means of the elementary $2 \mathrm{D}$ FE model are fitted to Ollendorf's mixing formula [6] and compared to previous results available in the literature.

\section{Proximity-effect complex permeability}

Let us define the reduced frequency $X$ as the ratio of a strand radius $r$ to the skin depth $\delta=\sqrt{2 /\left(\omega \sigma \mu_{0}\right)}$, i.e.

$$
X=\frac{r}{\delta}=\sqrt{f} \cdot r \sqrt{\pi \sigma \mu_{0}}
$$

with $f$ the working frequency (or pulsation $\omega=2 \pi f$ ), nonmagnetic strand with permeability $\mu_{0}=4 \pi 10^{-7} \mathrm{H} / \mathrm{m}$ and conductivity $\sigma$.

We obtain the reference proximity-effect complex permeability by solving an elementary FE model with a central cell (conductor plus insulation) and a single layer of surrounding cells [5]. A pure proximity-effect excitation is achieved by imposing a unit horizontal (or vertical) induction and zero net current through appropiate boundary conditions. By integrating over the central cell $\sigma^{-1} \boldsymbol{j} \boldsymbol{j}^{*} / 2$ with $\boldsymbol{j}$ the current density and $\omega \boldsymbol{b} \boldsymbol{b}^{*} / 2$ with $\boldsymbol{b}$ the flux density (r.m.s. values), we quantify the complex power absorved by the central cell (complex numbers in bold, $\imath$ imaginary unit). The complex proximity-effect reluctivity $\boldsymbol{\nu}_{\text {prox }}(X)=1 / \boldsymbol{\mu}_{\text {prox }}(X)$, at reduced frequency $X$, can be written in terms of two dimensionless coefficients [4]. Such a model with hexagonal packing $(r=0.1 \mathrm{~mm}$, fill factor $\lambda=0.69, X=2$ ) and imposed unit horizontal flux is represented in Fig. 1. The real and imaginary parts of the flux are depicted as well. Note that the packing type of litz-wire strands
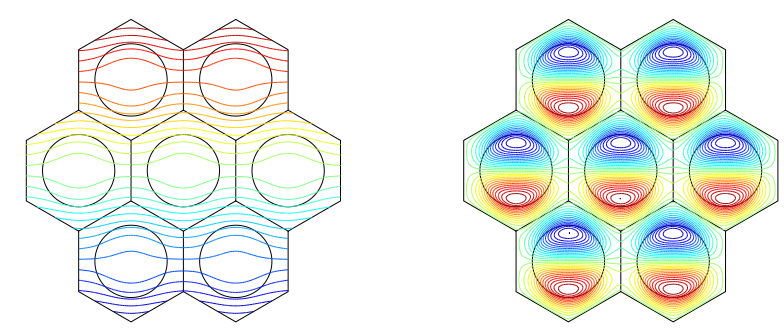

Figure 1. Elementary FE model with hexagonal packing and circular cross-section conductors. Proximity-effect flux pattern-real and imaginary components.

inside a bundle does not significantly affect the complex permeability [1], only the fill factor $\lambda$ (conductor to bundle area ratio) does Hexagonal packing is herein adopted.

As in Igarashi's studies [6], we choose the Ollendorff formula as ansatz to fit the reference FE solutions from the elementary problem [4]. It reads as follows

$$
\frac{\boldsymbol{\mu}_{\mathrm{prox}}}{\mu_{0}}=1+\frac{2 \boldsymbol{\eta}\left(\frac{\boldsymbol{\mu}_{\mathrm{w}}}{\mu_{0}}-1\right)}{2+(1-\boldsymbol{\eta})\left(\frac{\boldsymbol{\mu}_{\mathrm{w}}}{\mu_{0}}-1\right)},
$$

where $\boldsymbol{\mu}_{\mathrm{w}}$ is the macroscopic complex permeability of an iso- 
lated round wire [6]. It is given by

$$
\frac{\boldsymbol{\mu}_{\mathrm{w}}}{\mu_{0}}=\frac{\boldsymbol{J}_{1}(\boldsymbol{z})}{\boldsymbol{z} \boldsymbol{J}_{0}(\boldsymbol{z})-\boldsymbol{J}_{1}(\boldsymbol{z})},
$$

with $\boldsymbol{z}=(1-\boldsymbol{\imath}) X$ the complex reduced frequency, $\boldsymbol{J}_{0}$ and $\boldsymbol{J}_{1}$, the zero- and first-order Bessel functions. A corrected complex fill factor $\boldsymbol{\eta}$ is further introduced to match the FE results:

$$
\boldsymbol{\eta}=\lambda(1+\boldsymbol{\Theta}(\lambda) \boldsymbol{\Gamma}(\boldsymbol{z}))
$$

with $\boldsymbol{\Theta}(\lambda)=\sum_{0}^{n_{\Theta}} \boldsymbol{h}_{j} \lambda^{j}$ and $\boldsymbol{\Gamma}(\boldsymbol{z})=\sum_{0}^{n_{\Gamma}} \boldsymbol{g}_{j} \boldsymbol{z}^{j}$ the complex polynomials with orders $n_{\Theta}$ and $n_{\Gamma}$, respectively.

This approximation is fitted and then compared with Meeker's one [3], which is the most accurate in the literature. Indeed, a comparison with results in [7] and [1] can be found in [3]. For fair comparison, our fitting matches thus the range of frequencies and the order of the polynomials in [3], e.g. $X \in[0,30]$. Note that increasing the order of the polynomials in (4). The fitted coefficients $h_{j}$ and $g_{j}$ are displayed in Table 2.

\begin{tabular}{lr}
\hline $\boldsymbol{h}_{0}$ & $(-2.5159-\imath 0.1316) \times 10^{-2}$ \\
\hline $\boldsymbol{h}_{1}$ & $(1.9870+\imath 0.6130) \times 10^{-1}$ \\
\hline $\boldsymbol{h}_{2}$ & $(-5.1038-\imath 1.8358) \times 10^{-1}$ \\
\hline $\boldsymbol{h}_{3}$ & $(4.3864+\imath 1.4787) \times 10^{-1}$ \\
\hline \hline $\boldsymbol{g}_{0}$ & $(-1.1318+\imath 0.8916) \times 10^{-1}$ \\
\hline $\boldsymbol{g}_{1}$ & $(1.8429+\imath 3.0917) \times 10^{-2}$ \\
\hline $\boldsymbol{g}_{2}$ & $(2.1330-\imath 3.7990) \times 10^{-4}$ \\
\hline
\end{tabular}

Table 1. Coefficients $\boldsymbol{h}_{j}$ of $\boldsymbol{\Theta}(\boldsymbol{\lambda})$ with $n_{\Theta}=3$; and coefficients $\boldsymbol{g}_{j}$ of $\boldsymbol{\Gamma}(\boldsymbol{z})$ with $n_{\Gamma}=2$.
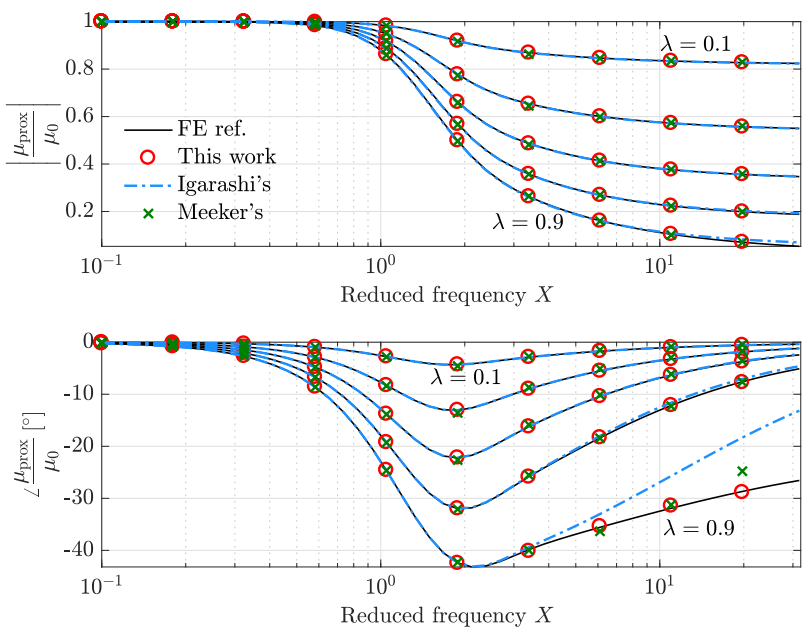

Figure 2. Relative complex permeability $\boldsymbol{\mu}_{\text {prox }} / \mu_{0}$ vs $X$ obtained with the reference FE problem, this work in (2), Igarashi's [6] and Meeker's expressions [3]. Results with fill factor $\lambda=0.1: 0.2: 0.9$.

The results of our new expression (2) are compared with Meeker's [3] and Igarashi's [6] approximations in Fig. 2. An excellent agreement is observed. We compute the relative $L_{2}$-norm error with regard to the reference FE solution of the three approximations. Meeker's approximate error is $1.3 \%$, our new expression (2) error is $0.3 \%$ and Igarashi's proximate error is $4.2 \%$. It is worth mentioning that we (2) and Igarashi [6] use the same accurate ansatz from Ollendorf's mixing formula, but with different fill factor. We incorporate a corrected complex fill factor $\Lambda$ while Igarashi's uses $\Lambda=\lambda$. The deviations of both Meeker's and Igarashi's expressions can be clearly seen from the phase response at high fill factor $(\lambda>0.7)$ and high frequencies $(X>3)$.

\section{Conclusions}

We have proposed a new complex frequency-dependent relutivity expression for homogenizing litz-wire windings in FEtype models. The agreement with the brute-force FE reference model is excellent in a wide frequency range, much wider than the practical range of working frequencies in classical electrotechnical problems, i.e. $X=r_{\mathrm{s}} / \delta \in[0,2]$. Most available formulae available $[1,6,3]$ cover accurately this practical frequency range. For the sake of comparison and in view of the current frequency trendin power devices, we have chosen to study the wide band $X=r_{\mathrm{s}} / \delta \in[0,30]$

\section{References}

[1] X. Nan and C. R. Sullivan, “An Equivalent Complex Permeability Model for Litz-Wire Windings," IEEE Trans. Ind. Appl., vol. 45, no. 2, pp. 854-860, 2009.

[2] G. Meunier, V. Charmoille, C. Guérin, P. Labie, Y. Maréchal, "Homogenization for Periodical Electromagnetic Structure: Which Formulation?," IEEE Trans. Magn., vol. 46, no. 8, pp. 3409-3412, 2010.

[3] D. C. Meeker, "An improved continuum skin and proximity effect model for hexagonally packed wires," J. Comput. Appl. Math., vol. 236, no. 18, pp. 4635-4644, 2012.

[4] J. Gyselinck, P. Dular, "Frequency-domain homogenization of bundles of wires in 2D magnetodynamic FE calculations," IEEE Trans. Magn., vol. 41, no. 1, pp. 1416-1419, 2005.

[5] K. Niyomsatian, J. Van den Keybus, R. V. Sabariego, J. Gyselinck, " Frequency-domain Homogenization for Impedance Characterization of Litz-Wire Transformers in 2-D Finite Element Models," 11th International Conference on Ecological Vehicles and Renewable Energies (EVER), Monte Carlo, Monaco, 6-8 April, 2016

[6] H. Igarashi, "Semi-analytical approach for finite-element analysis of multi-turn coil considering skin and proximity effects," IEEE Trans. Magn., vol. 53, no. 1, 2017.

[7] H. Rossmanith, M. Albach, J. Fischer, and A. Stadler, "Improved Characterization of the Magnetic Properties of Hexagonally Packed Wires," EPE J., vol. 22, no. 4, pp. 5$10,2012$. 